\title{
Infrared pupillometry to help predict neurological outcome for patients achieving return of spontaneous circulation following cardiac arrest: a systematic review protocol
}

\author{
Alex Monk ${ }^{1 *}$ (D) and Shashank Patil ${ }^{1,2}$
}

\begin{abstract}
Background: Despite advances in resuscitation care, mortality rates following cardiac arrest (CA) remain high. Between one-quarter (in-hospital CA) and two-thirds (out of hospital CA) of patients admitted comatose to intensive care die of neurological injury. Neuroprognostication determines an informed and timely withdrawal of life sustaining treatment (WLST), sparing the patient unnecessary suffering, alleviating family distress and allowing a more utilitarian use of resources. The latest Resuscitation Council UK (2015) guidance on post-resuscitation care provides the current multi-modal neuroprognostication strategy to predict neurological outcome. Its modalities include neurological examination, neurophysiological tests, biomarkers and radiology. Despite each of the current strategy's predictive modalities exhibiting limitations, meta-analyses show that three, namely PLR (pupillary light reflex), CR (corneal reflex) and N20 SSEP (somatosensory-evoked potential), accurately predict poor neurological outcome with low false positive rates. However, the quality of evidence is low, reducing confidence in the strategy's results. While infrared pupillometry (IRP) is not currently used as a prognostication modality, it can provide a quantitative and objective measure of pupillary size and PLR, giving a definitive view of the second and third cranial nerve activity, a predictor of neurological outcome.
\end{abstract}

Methods: The proposed study will test the hypothesis, "in those patients who remain comatose following return of spontaneous circulation (ROSC) after CA, IRP can be used early to help predict poor neurological outcome". A comprehensive review of the evidence using a PRISMA-P (2015) compliant methodology will be underpinned by systematic searching of electronic databases and the two authors selecting and screening eligible studies using the Cochrane data extraction and assessment template. Randomised controlled trials and retrospective and prospective studies will be included, and the quality and strength of evidence will be assessed using the Grading of Recommendation, Assessment and Evaluation (GRADE) approach.

Discussion: IRP requires rudimentary skill and is objective and repeatable. As a clinical prognostication modality, it may be utilised early, when the strategy's other modalities are not recommended. Corroboration in the evidence would promote early use of IRP and a reduction in ICU bed days.

Systematic review registration: PROSPERO CRD42018118180

Keywords: Cardiac arrest, Prognostication, Infrared pupillometry, Systematic review

\footnotetext{
* Correspondence: alex.monk@nhs.net

${ }^{1}$ Emergency Department, Chelsea and Westminster Hospital, 369 Fulham

Road, Chelsea, London SW10 9NH, England

Full list of author information is available at the end of the article
}

(c) The Author(s). 2019 Open Access This article is distributed under the terms of the Creative Commons Attribution 4.0 International License (http://creativecommons.org/licenses/by/4.0/), which permits unrestricted use, distribution, and reproduction in any medium, provided you give appropriate credit to the original author(s) and the source, provide a link to the Creative Commons license, and indicate if changes were made. The Creative Commons Public Domain Dedication waiver (http://creativecommons.org/publicdomain/zero/1.0/) applies to the data made available in this article, unless otherwise stated. 


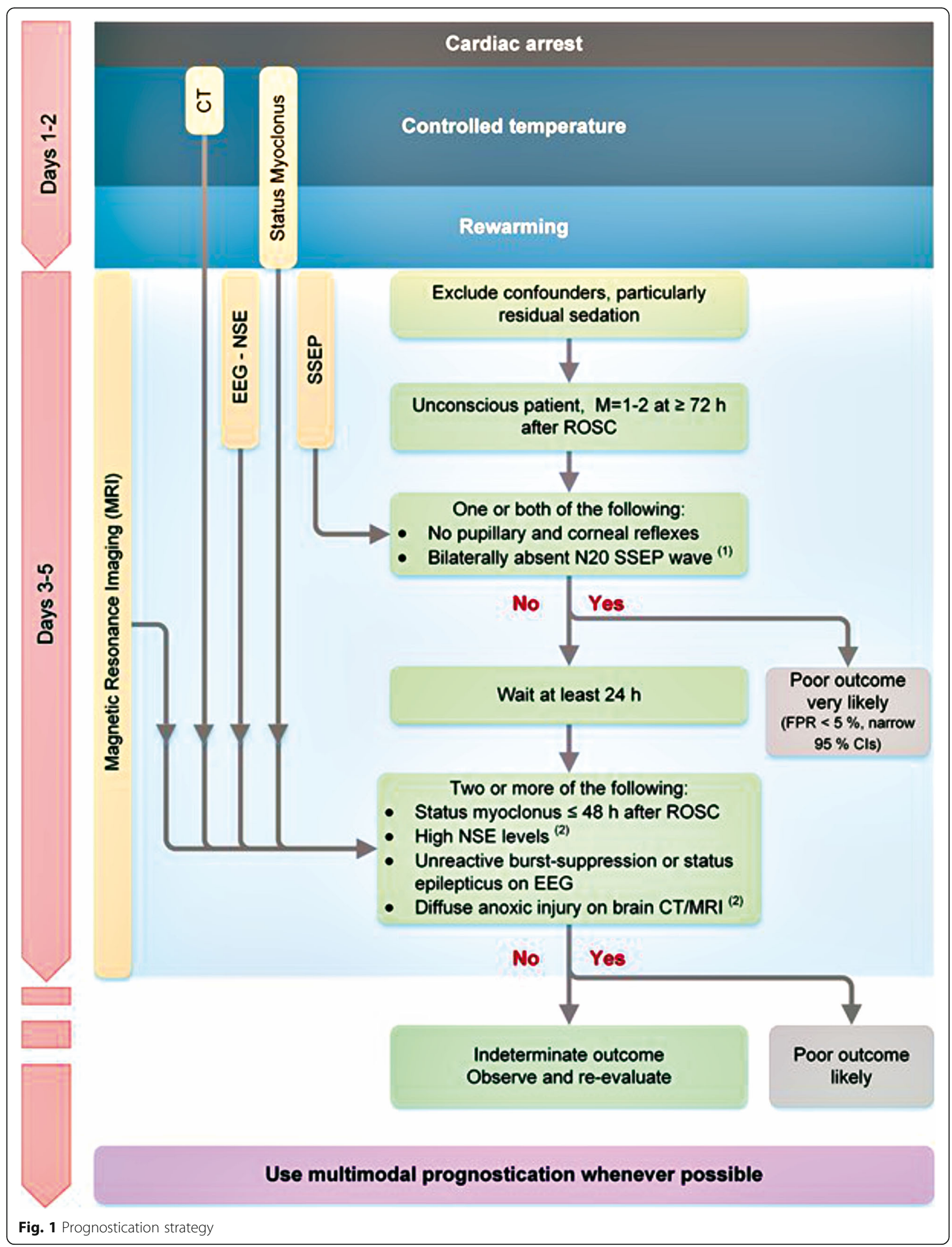




\section{Background}

Despite advances in resuscitation care, mortality rates following cardiac arrest (CA) remain high $[1,2]$. Between one-quarter (in-hospital CA) and two-thirds (out of hospital CA) of patients admitted comatose to intensive care, die of neurological injury [3], the majority as a result of withdrawal of life sustaining treatment (WLST) following a poor neuroprognosis [4]. While neuroprognosis determines an informed and timely WLST decision, it is complicated by a range of factors.

- On return of spontaneous circulation (ROSC) following cardiac arrest, management of postcardiac arrest syndrome [5] can be particularly challenging. Its components, brain injury, myocardial dysfunction, systemic ischaemia/reperfusion response and persistent precipitating pathology are inter-related, with observable benefits depending on optimisation of all.

- The latest Resuscitation Council UK (2015) guidance on post-resuscitation care [4] guards against post-ischaemic neuronal injury to maximise neurological recovery. The guidance includes adequate sedation and targeted temperature management (TTM), which can reduce the accuracy of prognostic modalities [6, 7].

- A systematic review of international data on prognostication modalities in comatose post-CA patients treated with TTM [8] gave rise to a multi-modal strategy for prognostication $[9,10]$, published jointly by ERC-ESICM (European Resuscitation Council and European Society of Intensive Care Medicine) [11, 12]. The strategy (Fig. 1) advises that prognostication is initially delayed $72 h$ after ROSC to allow rewarming and clearance of residual sedation [12].

- The prognostication strategy's modalities are differentiated in the guidance by their specificity, precision and robustness. The more robust modalities:

- bilaterally absent pupillary light reflex (PLR);

- corneal reflex (CR) and

- bilaterally absent N20 SSEP (somatosensoryevoked potential) wave;

are used first and their combined results used to prognosticate $[6,12]$. When a poor neurological outcome is not predicted to be 'very likely', less robust modalities are added after a further 24- $h$ delay $[6,12]$.

- Each predictive modality exhibits limitations. Studies show that some are subjective or prone to inconsistencies [13-16]. Others rely on specialist interpretation [17-20], are ill-defined [4] or are incompletely understood [6].
- Meta-analyses of the strategy's modalities show that PLR, CR and N20 SSEP predict poor neurological outcome with low false positive rates $[8,21]$. However, the primary studies provided 'low' to 'very low' quality of evidence $[4,6,7,21]$, reducing confidence in the prediction and therefore extending the observation period.

- The primary studies show that WLST is influenced by a self-fulfilling prophecy, a bias introduced when prognostic modalities are not blinded to the treating team $[4,6]$. As well as reducing the quality of the evidence, this bias reduces available evidence on delayed awakening (late recovery of consciousness following coma), which can affect $30 \%$ of post-CA patients [22]

The complex management of post-CA syndrome, neuroprotective measures' adverse impact on prognostication accuracy (sedation and TTM), consequent delays in prognostication (72 $\mathrm{h}$ and $24 \mathrm{~h}$ ), limitations of the individual modalities and unblinded observer bias, all present a challenge to early prediction of a poor neurological outcome.

The immediate consequence of a delayed poor neuroprognosis is futile treatment and resource wastage. Treatment is multifaceted, complex and costly. A 2015 study [23] (P.6) found 'a significant correlation between length of stay and cost, with a much longer length of stay in ICU and hospital for CPC (Glasgow-Pittsburgh Cerebral Performance Category [24]) 3-4 patients' (cf. CPC 1-2).

The intended benefit of treatment, quality adjusted life years (QALY), has a complex relationship with neurological status. Petrie et al showed that cost per QALY for ROSC post-CA, high-quality (CPC 1-2) survivors, at $£ 16,000$ [23], is well within the $£ 30,000$ UK (NICE) threshhold [25]. However, Petrie et al noted that 'a major determinant of cost for the CPC 1-2 group was the burden of cost of the non-survivors and CPC 3-4 patient group [23] (p.6). An aim of the proposed review is earlier neuroprognosis in CPC 3-4 patients, which will reduce this cost burden.

In principle, improving the current multi-modal prognostication strategy to enable earlier prediction of poor neurological outcome would allow a more utilitarian resource allocation, reduce futile treatment and lessen the healthcare opportunity cost resource depletion imposes. The usefulness of supplementary modalities that yield immediate results, require rudimentary operator skill and are amenable to easily repeatable tests is being investigated [20].

IRP is emerging as one such promising prognostic modality, with one recent study showing that it yields higher specificity and sensitivity than manual PLR measurement [26]. In IRP, infrared light is shone directly at 
the pupil and data is obtained through analysis of the reflected image. Characteristics of the PLR include, amplitude, latency constriction and dilatation velocity. This provides a direct functional assessment of the second and third cranial nerves, a predictor of neurological outcome.

\section{Objectives}

The proposed study will comprehensively review the evidence to determine whether the early use of IRP would help predict neurological outcome in comatose patients who achieve ROSC following CA. Questions of particular interest include the following:

Timeliness - Can IRP be used early in the prognostication strategy to inform an earlier WLST decision?

Specificity - Can IRP reduce the risk of falsely pessimistic prediction, reducing the lack of confidence that increases observation periods and inflates cost? Sensitivity - Can IRP reduce the incidence of delayed neuroprognoses, reducing ICU bed days?

Primary hypothesis - In those patients who remain comatose following ROSC after CA, IRP can be used early to help predict poor neurological outcome.

\section{Methods}

The design of the systematic review will follow the PRISMA-P 2015 checklist [27] (Additional file 1). The systematic review protocol is registered with PROSPERO under ID 'CRD42018118180' and is provided in Additional file 2 .

\section{Eligibility criteria}

This systematic review will consider randomised controlled trials, systematic reviews and retrospective and prospective cohort studies with specific study characteristics. Study populations will include adults over the age of 18 who suffered a cardiac arrest; the intervention will be infrared pupillometry performed early in the prognostication strategy; the primary outcome measure is neurological outcome.

Studies will be excluded if their study populations included cardiac arrests of traumatic aetiology, pregnant women or paediatric cases. Case reports will also be excluded.

Only published studies written in English language will be considered. Year of publication will not form part of the exclusion criteria.

\section{Information sources}

A comprehensive and systematic search of the following electronic databases will inform this systematic review. The Healthcare Databases Advanced Search (HDAS), accessed through NICE, will be used as the interface through which, EMBASE, MEDLINE and CINAHL databases are searched. The Cochrane Database of Systematic Reviews (CDSR) will be searched. A search for completed systematic reviews within PROSPERO will also be carried out. The search will be expanded through direct contact with authors of works' pending publication, reference mining and citation searching of the related literature and hand searching of relevant journals.

\section{Search strategy}

The search strategy was defined and applied by a specialist search strategist (PB). HDAS has the advantage that a common syntax can be used to search EMBASE, MEDLINE and CINAHL, albeit using controlled vocabulary appropriate to each database. Natural language keyword searches include 'cardiac arrest', 'prognosis' and 'infrared pupillometry'. Boolean logic was used to combine search terms and allow extraction of potentially suitable abstracts. The completed search strategy for EMBASE, MEDLINE and CINAHL is provided in Additional file 3; this will be peer reviewed, using the Peer Review of Electronic Search Strategies (PRESS [28]) checklist, by an independent information specialist.

\section{Data management}

The extracted abstracts will be managed in HDAS.

\section{Study selection process and data collection process}

The study selection process will follow the PRISMA 2009 flow diagram from the PRISMA statement [27]. Extracted abstracts will be screened for duplicates before review by two authors AM and SP. Abstracts that are not excluded during initial review will undergo full text screening by each author independently.

A modified version of the Cochrane data extraction and assessment template [29] (Additional file 4) will be used for full text screening and data extraction. Within this, any reasons for exclusion will be noted. Variable and outcome data will be extracted in the same template. When the required data is not specified in a study's full text, all reasonable attempts will be made to contact the author and the content of any correspondence will be documented.

\section{Data items}

The following variables will be extracted from eligible studies: patient age and gender, outcome of cardiac arrest and quantitative values of infrared pupillometry.

\section{Outcomes and prioritisation}

The main outcome measure to be extracted from eligible studies is neurological outcome. Additional outcomes include the number of ICU bed days and survival at discharge. 
Table 1 Patient outcomes

\begin{tabular}{lll}
\hline & Poor outcome & Good outcome \\
\hline Very likely' poor prognosis & PP & PG \\
& WLST & False positive \\
No 'very likely' poor prognosis & GP & GG \\
& False negative & $£ 16 \mathrm{k} / \mathrm{QALY}$ Survivors \\
\hline
\end{tabular}

\section{Risk of bias in individual studies}

Risk-of-bias assessments will be made independently by two authors AM and SP. For all RCTs, we will use the Cochrane risk-of-bias (RoB) tool [30] and for all nonrandomised studies, the ROBINS-1 tool [31].

The quality and strength of evidence will be assessed using the Grades of Recommendation, Assessment and Evaluation (GRADE) approach [32].

\section{Internal validity}

Inter-reviewer agreement in study inclusion, data extraction and risk-of-bias assessments will be maximised by piloting the data extraction form and risk-of-bias tools prior to use in the systematic review. Clear usage instructions will include guidance on consistency of input styles and documentation of missing information [29].

Inter-reviewer agreement will be measured using the kappa statistic for the initial study screening, data extraction and risk-of-bias assessment.

A third reviewer will independently review the studies that elicit disagreement between AM and SP in study inclusion, data extraction or risk-of-bias assessments. Disagreements will be discussed between all three reviewers at regular team meetings. Each disagreement will be interrogated with reference to the decision rules and guidance on the use of the data extraction tool and riskof-bias assessments. The outcome and reason for the initial disagreement will be recorded.

\section{Synthesis and meta-biases}

This review aims to establish the association between IRP values that indicate a 'very likely' poor prognosis and patients with poor neurological outcome (defined as CPC 3-5).

IRP data will be correlated with patient outcome (Table 1) and employed in odds ratios to determine IRP's effect as an additional modality within the neuroprognostic algorithm. Taking account of the results from all studies, we will calculate aggregated estimates of the effect of intervention, together with $p$ values, means, confidence intervals and value ranges.

A Forest plot will be used to graphically represent the size of the effect seen in individual studies and the summarised effect.

Of particular interest are IRP's specificity, $\mathrm{PP} /(\mathrm{PP}+$ $\mathrm{PG})$; sensitivity, $\mathrm{PP} /(\mathrm{PP}+\mathrm{GP})$; false positive rate, $\mathrm{PG} /$ $(\mathrm{PP}+\mathrm{PG})$ and its false negative rate, $\mathrm{GP} /(\mathrm{GP}+\mathrm{GG})$.
Variation will be checked for between studies (heterogeneity) using Cochran's Q and other I-square statistics [33]. If significant heterogeneity is found, we will apply the random-effects model [34]. To identify sources of heterogeneity and adjust for them, sub-group analysis will be performed and meta-regression will be used to identify the influence co-variates have on the overall effect.

Homogeneity will be addressed using the fixed effects model [34].

\section{Confidence in cumulative evidence}

The quality and strength of evidence will be assessed using the Grades of Recommendation, Assessment and Evaluation (GRADE) approach [32].

\section{Discussion}

The current multi-modal neuroprognostication strategy advises that following ROSC after CA, clinicians wait 72 $\mathrm{h}$ to allow rewarming and clearance of sedation before prognosticating. A poor neuroprognosis prompts WLST, any usable strategy must minimise the risk of falsely pessimistic predictions, the false positive rate.

Despite each of the current strategy's predictive modalities exhibiting limitations, meta-analyses of the modalities show that PLR, CR and N20 SSEP predict poor neurological outcome with low false positive rates $[8,21]$. However, the quality of evidence is low, thereby reducing confidence in the strategy's results.

In the clinical utilisation of the strategy, false positive risk is mitigated by extending observation periods and utilising additional modalities [6]. Concomitantly, risk aversion increases the false negative rate (lack of a poor prognosis preceding a poor neurological outcome). The costs of risk mitigation [23] can be reduced through earlier prognostication using greater-specificity/greatersensitivity modalities that are objective, repeatable and can be deployed early in the strategy using readily available expertise. Greater specificity will increase confidence in predictions of poor neurological outcome, obviating the need to extend the observation period, and greater sensitivity will reduce the false negative rate.

IRP's characteristics, objectivity, repeatability and rudimentary operation make it a promising candidate for review. The proposed study will comprehensively review the evidence for IRP and determine whether it will help predict a poor neurological outcome post-CA.

\section{Supplementary information}

Supplementary information accompanies this paper at https://doi.org/10 1186/s13643-019-1209-z.

Additional file 1. PRISMA-P (Preferred Reporting Items for Systematic review and Meta-Analysis Protocols) 2015 checklist: recommended items to address in a systematic review protocol. 
Additional file 2. PROSPERO registration document.

Additional file 3. Search Strategy on EMBASE, MEDLINE and CINAHL.

Additional file 4. Cochrane public health group data extraction and assessment template.

\section{Abbreviations}

AM: Alex Monk; CA: Cardiac arrest; CDSR: Cochrane Database of Systematic Reviews; CPC: Glasgow-Pittsburgh Cerebral Performance Category; CR: Corneal reflex; CT: Computerised tomography;

EEG: Electroencephalography; ERC: European Resuscitation Council; ESICM: European Society of Intensive Care Medicine; FPR: False positive rate; GG: No poor neuroprognosis-good neurological outcome; GP: No poor neuroprognosis-poor neurological outcome; GRADE: Grades of Recommendation, Assessment and Evaluation; HDAS: Healthcare Databases Advanced Search; ICU: Intensive care unit; IRP: Infrared pupillometry; MeSH: Medical Subject Headings; MRI: Magnetic resonance imaging; NICE: National Institute for Health and Care Excellence; NSE: Neuron-specific enolase; PB: Phillip Barlow; PG: Poor neuroprognosis-good neurological outcome; PLR: Pupillary light reflex; PP: Poor neuroprognosis-poor neurological outcome; PRESS: Peer Review of Electronic Search Strategies; PRISMA-P: Preferred reporting items for systematic review and meta-analysis protocols; QALY: Quality adjusted life year; RCT: randomised controlled trial; RoB: Risk of bias; Robins-l: Risk of bias in non-randomised studies of interventions; ROSC: Return of spontaneous circulation; SP: Shashank Patil; SSEP: Somatosensory-evoked potential; TTM: Targeted temperature management; WLST: Withdrawal of life sustaining treatment

\section{Acknowledgements}

We would like to acknowledge the contribution of Mr. Philip Barlow who assisted in developing the search strategy.

\section{Authors' contributions}

AM prepared the manuscript for the systematic review and provided input on its focus and design. SP conceived the systematic review and provided definitive and final guidance on the manuscript. Both authors read and approved the final manuscript.

\section{Funding}

Not applicable.

\section{Availability of data and materials}

All data pertaining to this review protocol can be found within the body of text or in the supplementary additional files.

\section{Ethics approval and consent to participate}

Ethics approval and consent are waived.

\section{Consent for publication}

Not applicable.

\section{Competing interests}

The authors declare that they have no competing interests.

\section{Author details}

${ }^{1}$ Emergency Department, Chelsea and Westminster Hospital, 369 Fulham Road, Chelsea, London SW10 9NH, England. 'Kings College London, Room No 213, St Thomas' House, Westminster Bridge Road, London SE1 7EH, England.

Received: 3 April 2019 Accepted: 22 October 2019

Published online: 25 November 2019

\section{References}

1. Perkins PG. Review of the first 5 years and forward strategy. Out of Hospital Cardiac Arrest Outcomes (OHCAO) Registry. The University of Warwick. 2018. https://aace.org.uk/wp-content/uploads/2018/05/Out-of-Hospital-CardiacArrest-Outcomes-OHCAO-Registry-57904-CTU-Report-final.pdf. Accessed 17 Feb 2019
2. Nolan JP, Soar J, Smith GB, Gwinnutt C, Parrott F, Power S, et al. Incidence and outcome of in-hospital cardiac arrest in the United Kingdom National Cardiac Arrest Audit. Resuscitation. 2014;85(8):987-92.

3. Laver S, Farrow C, Turner D, Nolan J. Mode of death after admission to an intensive care unit following cardiac arrest. Intens Care Med. 2004; 30(11):2126-8.

4. Sandroni C, D’Arrigo S, Nolan J. Prognostication after cardiac arrest. Crit Care. 2018:22:150.

5. Nolan JP, Neumar RW, Adrie C, Aibiki M, Berg RA, Bottiger BW, et al. Postcardiac arrest syndrome: epidemiology, pathophysiology, treatment, and prognostication. A Scientific Statement from the International Liaison Committee on Resuscitation; the American Heart Association Emergency Cardiovascular Care Committee; the Council on Cardiovascular Surgery and Anesthesia the Council on Cardiopulmonary, Perioperative, and Critical Care the Council on Clinical Cardiology. Resuscitation. 2008;79(3):350-79.

6. Nolan JP, Deakin C, Lockey A, Perkins G, Soar J. Post-resuscitation care, Guidelines 2015. Resuscitation Council (UK). 2015.

7. Samaniego EA, Mlynash M, Caulfield AF, Eyngorn I, Wijman CAC. Sedation confounds outcome prediction in cardiac arrest survivors treated with hypothermia. Neurocritical Care. 2011;15(1):113-9.

8. Sandroni C, Cavallaro F, Callaway CW, D'Arrigo S, Sanna T, Kuiper MA, et al. Predictors of poor neurological outcome in adult comatose survivors of cardiac arrest: a systematic review and meta-analysis. Part 2: patients treated with therapeutic hypothermia. Resuscitation. 2013;84(10):1324-38.

9. Hazinski MF, Nolan JP, Aicken R, Bhanji F, Billi JE, Callaway CW, et al. Part I. Executive summary: 2015 International Consensus on Cardiopulmonary Resuscitation and Emergency Cardiovascular Care Science with Treatment Recommendations. Resuscitation. 2015;95:e1-e32.

10. Callaway CW, Soar J, Aibiki M, Böttiger BW, Brooks SC, Deakin CD, et al. Part 4: advanced life support: 2015 International Consensus on Cardiopulmonary Resuscitation and Emergency Cardiovascular Care Science With Treatment Recommendations. Resuscitation. 2015:95:e71-e122.

11. Nolan JP, Soar J, Cariou A, Cronberg T, Moulaert VR, Deakin CD, et al. European Resuscitation Council and European Society of Intensive Care Medicine Guidelines for Post-resuscitation Care 2015: Section 5 of the European Resuscitation Council Guidelines for Resuscitation 2015. Resuscitation. 2015;95:202-22.

12. Sandroni C, Cariou A, Cavallaro F, Cronberg T, Friberg H, Hoedemaekers C, et al. Prognostication in comatose survivors of cardiac arrest: an advisory statement from the European Resuscitation Council and the European Society of Intensive Care Medicine. Resuscitation. 2014;85(12):1779-89.

13. Du R, Meeker M, Bacchetti P, Larson MD, Holland MC, Manley GT. Evaluation of the portable infrared pupillometer. Neurosurgery. 2005;57(1):198-203.

14. Couret D, Boumaza D, Grisotto C, Triglia T, Pellegrini L, Ocquidant P, et al. Reliability of standard pupillometry practice in neurocritical care: an observational, double-blinded study. Critical Care. 2016;20:99.

15. Mlynash M, Buckwalter MS, Okada A, Caulfield AF, Venkatasubramanian C, Eyngorn I, et al. Serum neuron-specific enolase levels from the same patients differ between laboratories: assessment of a prospective postcardiac arrest cohort. Neurocritical Care. 2013;19(2):161-6.

16. Rundgren $\mathrm{M}$, Cronberg $\mathrm{T}$, Friberg $\mathrm{H}$, Isaksson A. Serum neuron specific enolase - impact of storage and measuring method. BMC Research Notes. 2014;7:726.

17. English WA, Giffin NJ, Nolan JP. Myoclonus after cardiac arrest: pitfalls in diagnosis and prognosis. Anaesthesia. 2009:64(8):908-11.

18. Aicua Rapun I, Novy J, Solari D, Oddo M, Rossetti AO. Early Lance-Adams syndrome after cardiac arrest: prevalence, time to return to awareness, and outcome in a large cohort. Resuscitation. 2017;115:169-72.

19. Elmer J, Rittenberger JC, Faro J, Molyneaux BJ, Popescu A, Callaway CW, et al. Clinically distinct electroencephalographic phenotypes of early myoclonus after cardiac arrest. Annals of Neurology. 2016;80(2):175-84.

20. Zandbergen EGJ, Hijdra A, de Haan RJ, van Dijk JG. Ongerboer de Visser BW, Spaans F, et al. Interobserver variation in the interpretation of SSEPs in anoxic-ischaemic coma. Clinical Neurophysiology. 2006;117(7):1529-35.

21. Golan E, Barrett K, Alali AS, Duggal A, Jichici D, Pinto R, et al. Predicting neurologic outcome after targeted temperature management for cardiac arrest: systematic review and meta-analysis. Critical Care Medicine. 2014 42(8):1919-30.

22. Paul M, Bougouin W, Geri G, Dumas F, Champigneulle B, Legriel S, et al. Delayed awakening after cardiac arrest: prevalence and risk factors in the Parisian registry. Intensive Care Medicine. 2016;42(7):1128-36. 
23. Petrie J, Easton S, Naik V, Lockie C, Brett SJ, Stumpfle R. Hospital costs of out-of-hospital cardiac arrest patients treated in intensive care; a single centre evaluation using the national tariff-based system. BMJ Open. 2015; 5(4):e005797.

24. Jennett B, Bond M. Assessment of outcome after severe brain damage. Lancet. 1975;1(7905):480-4.

25. National Institute for Clinical Excellence (NICE). Incorporating health economics. In: Methods for the development of NICE public health guidance (third edition). NICE. 2012. https://www.nice.org.uk/process/pmg4/ chapter/incorporating-health-economics. Accessed 17 Feb 2019

26. Solari D, Rossetti AO, Carteron L, Miroz JP, Novy J, Eckert P, et al. Early prediction of coma recovery after cardiac arrest with blinded pupillometry. Ann Neurol. 2017;81(6):804-10

27. Liberati A, Altman DG, Tetzlaff J, Mulrow C, Gotzsche PC, loannidis JPA, et al. The PRISMA statement for reporting systematic reviews and meta-analyses of studies that evaluate health care interventions: explanation and elaboration. Ann Int Med. 2009;151(4):W-65-94.

28. Sampson M, McGowan J, Cogo E, Grimshaw J, Moher D, Lefebvre C. An evidence-based practice guideline for the peer review of electronic search strategies. J Clin Epidemiol. 2009;62(9):944-52.

29. The Cochrane Collaboration. The Cochrane public health group. Data extraction and assessment template. 2011. https://ph.cochrane.org/sites/ph. cochrane.org/files/public/uploads/CPHG\%20Data\%20extraction\%2 Otemplate_0.docx. Accessed 17 Feb 2019

30. The Cochrane Collaboration. Cochrane methods bias. Cochrane risk of bias tool for randomized controlled trials. http://methods.cochrane.org/bias/ assessing-risk-bias-included-studies

31. Sterne JAC, Higgins J, Reeves BC. A Cochrane risk of bias assessment tool: for non-randomised studies of interventions (ACROBAT-NRSI). Version 1.0.0 24 edn; 2014. http://www.riskofbias.info.

32. The Cochrane Collaboration. Cochrane handbook of systematic review of interventions. Version 5.1.0; 12.2.1 The GRADE approach. http://training. cochrane.org/handbook. Accessed 17 Feb 2019

33. Higgins JPT, Thompson S, Deeks JJ, Altman DG. Measuring inconsistency in meta-analyses. BMJ. 2003;327(7414):557-60.

34. Hunter JE, Schmidt FL. Methods of meta-analysis: correcting error and bias in research findings: Sage; 2004

\section{Publisher's Note}

Springer Nature remains neutral with regard to jurisdictional claims in published maps and institutional affiliations.

Ready to submit your research? Choose BMC and benefit from:

- fast, convenient online submission

- thorough peer review by experienced researchers in your field

- rapid publication on acceptance

- support for research data, including large and complex data types

- gold Open Access which fosters wider collaboration and increased citations

- maximum visibility for your research: over $100 \mathrm{M}$ website views per year

At $\mathrm{BMC}$, research is always in progress.

Learn more biomedcentral.com/submissions 